\title{
Entwicklungsstörungen des Sprechens oder der Sprache nach ICD-11
}

\author{
Christine M. Freitag ${ }^{1}$, Michelle Noterdaeme², Kristin Snippe ${ }^{3}$, Petra Schulz4, Ziyon Kim¹ und \\ Karoline Teufel ${ }^{1}$
}

\author{
Klinik für Psychiatrie, Psychosomatik und Psychotherapie des Kindes- und Jugendalters, Autismus-Therapie- und \\ Forschungszentrum, Universitätsklinikum Frankfurt, Goethe-Universität Frankfurt am Main \\ 2 Klinik für Kinder- und Jugend psychiatrie und -psychotherapie, Fachklinik Josefinum Augsburg \\ 3 Praxis für Logopädie, Berlin \\ 4 Institut für Psycholinguistik und Didaktik der deutschen Sprache, Goethe-Universität Frankfurt am Main
}

\begin{abstract}
Zusammenfassung: Sprach- und Sprechstörungen kommen bei zahlreichen Kindern vor und werden in der ICD-11 analog zur ICD-10 als Entwicklungsstörungen im Kapitel 6 (Psychische, Verhaltens- und Entwicklungsstörungen) klassifiziert. International sind bislang die ICD-10-Kriterien nicht von allen Professionen, die sich mit Sprach- und Sprechstörungen klinisch oder im Rahmen der Forschung beschäftigen, akzeptiert. Sie werden einerseits als zu wenig differenziert hinsichtlich der unterschiedlichen Sprachkomponenten vonseiten der Linguistik, Sprachtherapie oder Logopädie erlebt. Zum anderen wird die unklare Abgrenzung organisch bedingter Sprach- und Sprechprobleme von der Sprachentwicklungsstörung vonseiten der Medizin teilweise kritisch bewertet. In dem vorliegenden Artikel wird deshalb einerseits die Klassifikation von Sprach- und Sprechproblemen und -störungen in der ICD-11 im Vergleich zur ICD-10 vorgenommen. Wesentlich erscheint hier die in der ICD-11 neu eingeführte Differenzierung in „primäre“ und „sekundäre“ Neuroentwicklungsstörungen. Zum anderen erfolgt aber auch eine Auseinandersetzung mit dem DSM-5 sowie anderen Klassifikationsvorschlägen vonseiten der englischsprachigen Sprachtherapie (CATALISE-2) und der deutschsprachigen Pädaudiologie (,phonologische Wahrnehmungsstörung“) sowie der Vorschlag einer Ergänzung der aktuellen ICD-11-Klassifikation hinsichtlich konkreter sprachlicher Einschränkungen bei einem Kind mit Sprachentwicklungsstörung, basierend auf einer ausführlichen Diagnostik. Wir hoffen, mit dem Artikel so den Weg für eine berufsübergreifende Klassifikation von Sprach- und Sprechstörungen nach ICD-11 zu bahnen, damit perspektivisch alle Berufsgruppen, die Diagnostik und Therapie der betroffenen Personen anbieten, eine vergleichbare Terminologie verwenden. Diese vergleichbare Terminologie soll sowohl die klinische Versorgung verbessern als auch die unterschiedlichen Forschungsansätze und -richtungen vergleichbarer machen.
\end{abstract}

Schlüsselwörter: Sprechen, Sprache, Klassifikation, Sprachstörung, Sprechstörung

\section{Developmental Speech and Language Disorders According to ICD-11}

Abstract: In ICD-11, similar to ICD-10, speech and language disorders are classified as neurodevelopmental disorders, which are part of ICD-11 Chapter 6 (Mental, Behavioural and Neurodevelopmental Disorders). The ICD-10 criteria were not well accepted by many professionals in research and clinic who work with children with speech and language disorders. Especially linguists and speech and language therapists see ICD-10 as too crude and lacking specification of individual language problems. Medical professions in turn criticize the missing aspect of organically caused speech and language problems. This paper presents the classification of speech and language problems or disorders according to ICD-11 compared to ICD-10. One essential aspect lies in the differentiation between "primary" and "secondary" neurodevelopmental disorders. In addition, we compare and discuss other recent classification approaches, such as DSM-5, CATALISE-2, and the classification "Auditory Processing Disorder" by pediatric audiologists. We present a classification approach based on ICD-11, supplemented by an additional specification of the respective impaired speech or language area in the individual child and based on a thorough speech and language assessment. We thus hope to pave the path for an interdisciplinary classification of speech and language disorders according to ICD-11, our aim being to establish a common terminology that can be used by all professions. We expect this common terminology to improve clinical care and to allow for the integration and comparability of speech- and language-related research efforts.

Keywords: speech, language, classification, speech and language disorder 


\section{Einleitung}

Sprach- und Sprechstörungen werden in der ICD-11 (letzte Version: https://icd.who.int/browse11/l-m/en) analog zur ICD-10 als Entwicklungsstörungen (ICD-10: F8) klassifiziert (Weltgesundheitsorganisation, 1994). In der ICD-11 werden sie unter den Neuroentwicklungsstörungen (,neurodevelopmental disorders“, $\left.06^{1}\right)$ gelistet. In das entsprechende Kapitel 06 sind zusätzlich Störungen wie Intelligenzminderung, Autismus-Spektrum-Störung, Lernstörungen, motorische Koordinationsstörung, Aufmerksamkeitssdefizit-/Hyperaktivitätsstörung und die stereotype Bewegungsstörung eingruppiert.

Um die Logik der Klassifikation von Sprach- und Sprechstörungen sowie die entsprechenden Diagnosekriterien nach ICD-11 nachvollziehen zu können, ist deshalb ein Vergleich sowohl mit den allgemeinen Kriterien einer Neuroentwicklungsstörung als auch mit den Diagnosekriterien der anderen Störungen, die in Kapitel 06 klassifiziert sind, hilfreich.

Die wesentlichen Charakteristika der Neuroentwicklungsstörungen nach ICD-11 sind folgende: Es handelt sich um Verhaltens- oder kognitive Störungen, die sich im Laufe der Entwicklung zeigen und zu deutlichen Schwierigkeiten in der Aneignung und in der Anwendung spezifischer intellektueller, motorischer, sprachlicher oder sozialer Funktionen führen. In Abgrenzung $\mathrm{zu}$ anderen psychischen Störungen, wie z. B. Schizophrenie oder bipolare Störung, wird der Entwicklungsaspekt betont, d.h. ein Beginn in der (frühen) Kindheit ist wesentlich für Neuroentwicklungsstörungen.

Die Kategorie 6E60 „sekundäre Neuroentwicklungsstörungen" wird explizit davon abgegrenzt. Letztere sind durch folgende Aspekte charakterisiert: Syndrome, die zwar Charakteristika von Neuroentwicklungsstörungen, aber nicht die vollen diagnostischen Kriterien der genuinen Neuroentwicklungsstörungen aufweisen. Die entsprechenden Syndrome müssen eine direkte Konsequenz der zugrunde liegenden organischen Erkrankung sein (z.B. autistische Verhaltensweisen bei Rett-Syndrom, Aggression und Selbstverletzung bei Lesch-Nyhan-Syndrom, Sprachentwicklungsprobleme bei Williams-Syndrom). Wenn eine entsprechende organisch-genetische Diagnose vorliegt und die Symptome so stark ausgeprägt sind, dass sie behandlungsbedürftig sind, dann soll die Zusatzdiagnose einer sekundären Neuroentwicklungsstörung zusätzlich zu der zugrunde liegenden organischen Erkrankung gestellt werden.

Die o.g. Differenzierung zwischen „primären“ und sekundären Neuroentwicklungsstörungen gilt demnach auch für die Sprach- und Sprechstörungen. Nach ICD-11 dürfen als Entwicklungsstörungen des Sprechens oder der Sprache also nur Erkrankungen klassifiziert werden, die einerseits die vollständigen Diagnosekriterien erfüllen (s.u.) und andererseits nicht ausschließlich durch eine organische Erkrankung einschließlich eines genetischen Syndroms begründet sind (Tabelle 1). Hinsichtlich der Klassifikation von Sprach- und Sprechproblemen, die vollständig durch eine organische Erkrankung verursacht sind, gibt es in der ICD-11 eine eigene Kategorie: 6E60.0 Sekundäres Sprech- oder Sprachsyndrom („secondary speech or language syndrome“). Die diagnostischen Kriterien lauten entsprechend: Es liegt ein Syndrom vor, bei dem sich einzelne Aspekte von Sprach- und Sprechstörungen zeigen, die jedoch nicht die vollen diagnostischen Kriterien einer Entwicklungsstörung der Sprache und des Sprechens erfüllen. Die Symptome sollen eine direkte pathophysiologische Konsequenz der ihnen zugrunde liegenden Gesundheitseinschränkung sein, die ihrerseits nicht unter den psychischen und Verhaltensstörungen klassifiziert sein darf (ICD-11, Kapitel 06). Ein Hinweis auf beispielhafte mögliche zugrunde liegende Erkrankungen erfolgt hinsichtlich Erkrankungen des Nervensystems, sensorischer Einschränkungen, Hirnverletzung oder Infektionen. Die Diagnose eines sekundären Sprech- oder Sprachsyndroms soll dann in Ergänzung zu der diagnostizierten zugrunde liegenden organischen Erkrankung (einschließlich genetischer Syndrome) vergeben werden.

In Tabelle 2 sind in der letzten Zeile 6 zahlreiche Ausschlussdiagnosen der Entwicklungsstörungen des Sprechens oder der Sprache (ICD-11: 6A01) aufgeführt, die einerseits beschreibend sind (wie 6E60.0: Sekundäres Sprech- oder Sprachsyndrom) und als Zusatzdiagnosen bei organischen Grunderkrankungen vergeben werden können, andererseits auf eine spezifische Ätiologie von Sprachoder Sprechproblemen hinweisen können, wie z. B. 8A62.2: Erworbene epileptische Aphasie, AB50-57: Hörstörungen oder LD2H: syndromische genetische Taubheit.

Im Folgenden vergleichen wir zunächst ICD-11 und ICD10 hinsichtlich der "primären“ Entwicklungsstörungen des Sprechens und der Sprache. Da die ICD-11 der US-amerikanischen Klassifikation DSM-5 in einigen Punkten ähnlich ist, wird auch die amerikanische Klassifikation DSM-5 erwähnt, um die Leser_innen, die DSM-5 nicht regelhaft anwenden, entsprechend zu informieren (American Psychiatric Association, 2020). Zusätzlich wird im englischen Sprachraum derzeit eine davon abgegrenzte Klassifikation von Sprach- und Sprechstörungen (CATALISE-2) diskutiert, die auch von Linguistinnen im deutschsprachigen Raum aufgegriffen wurde (Kauschke \& Vogt, 2019). Dies erscheint insbesondere deshalb wichtig, da im klinischen 
Tabelle 1. Allgemeine ICD-11-Kriterien der Entwicklungsstörungen des Sprechens und der Sprache

\begin{tabular}{|c|c|c|}
\hline Bereiche & Einschlusskriterien & Ausschlusskriterien \\
\hline Entwicklung & Beginn in der frühen kindlichen Entwicklung & $\begin{array}{l}\text { Es darf keine Periode mit unauffälligen, altersge- } \\
\text { mäßen Sprach- oder Sprechfertigkeiten vorliegen }\end{array}$ \\
\hline Sprache & $\begin{array}{l}\text { Verständnisschwierigkeiten } \\
\text { Produktionsschwierigkeiten } \\
\text { Probleme im funktionellen Einsatz der Sprache } \\
\text { zur Kommunikation }\end{array}$ & \\
\hline Sprechen & Artikulations- und Redeflussstörung & \\
\hline Altersbezug & $\begin{array}{l}\text { Sprach- und Sprechfertigkeiten liegen außerhalb } \\
\text { der normalen Altersvariabilität }\end{array}$ & $\begin{array}{l}\text { Sprach- und Sprechfertigkeiten liegen innerhalb } \\
\text { der normalen Altersvariabilität }\end{array}$ \\
\hline Nonverbale Intelligenz & $\begin{array}{l}\text { Sprach- oder Sprechfertigkeiten liegen außerhalb } \\
\text { der normalen kognitiven Variabilität }\end{array}$ & $\begin{array}{l}\text { Sprach- oder Sprechfertigkeiten liegen innerhalb } \\
\text { der normalen kognitiven Variabilität }\end{array}$ \\
\hline Soziale Hintergründe & Dürfen kein wesentlicher Grund sein & $\begin{array}{l}\text { Soziale Deprivation und fehlende Förderung er- } \\
\text { klärt die Symptome }\end{array}$ \\
\hline $\begin{array}{l}\text { Kulturelle/regionale/ethnische } \\
\text { Hintergründe }\end{array}$ & Dürfen kein wesentlicher Grund sein & $\begin{array}{l}\text { Zweisprachigkeit, Zweitsprache, Migration und } \\
\text { lokaler Dialekt bzw. (sub)kulturspezifische Aus- } \\
\text { drucksweise erklärt die Symptome }\end{array}$ \\
\hline Somatische komorbide Störungen & Dürfen die Symptomatik nicht vollständig erklären & $\begin{array}{l}\text { Somatische Ursachen (anatomische oder neuro- } \\
\text { logische Befunde) erklären die Symptomatik voll- } \\
\text { ständig }\end{array}$ \\
\hline Psychische komorbide Störungen & Dürfen vorkommen & Kein Ausschlussgrund \\
\hline
\end{tabular}

Bereich in Deutschland zahlreiche Professionen (Logopäd_ innen, Sprachtherapeut_innen, Linguist_innen, Kinderund Jugendpsychiater_innen, Kinder- und Jugendlichenpsychotherapeut_innen, Pädaudiolog_innen, Kinder- und Jugendärzt_innen) einerseits im Rahmen der Forschung, andererseits klinisch mit Patient_innen arbeiten, die Sprach- und Sprechprobleme aufweisen, es aber leider keine professionsübergreifende, von allen anerkannte Klassifikation dieser Symptome und Erkrankungen gibt. Da die ICD in der deutschen Sozialgesetzgebung das grundlegende Klassifikationssystem darstellt, wird abschließend ein klinisch-praktisches Vorgehen hinsichtlich der Umsetzung und Ergänzung der ICD-11-Klassifikation im deutschen Gesundheits- und Sozialsystem skizziert, das die Abgrenzung ,primär" und „sekundär" aus der ICD-11 aufgreift.

\section{Von ICD-10 zu ICD-11}

Hinsichtlich der Klassifikation von Sprach- und Sprechstörungen ergeben sich im Vergleich von ICD-10 zu ICD-11 die folgenden wesentlichen Änderungen (siehe Tabelle 2):

- Bei der Oberkategorie der ICD-11 6A01: Entwicklungsstörungen des Sprechens oder der Sprache (ESS) wurde die Bezeichnung ,umschrieben“ gestrichen. Damit wird dem Umstand Rechnung getragen, dass viele Kinder mit einer ESS zusätzlich auch weitere motorische oder kognitive Einschränkungen aufweisen.

- In der ICD-11 erfolgt eine Differenzierung in Sprechoder Sprachstörungen unter der Kategorie 6A01: Entwicklungsstörungen des Sprechens oder der Sprache (ESS). Dieser Logik folgt, dass einerseits die Redeflussstörung (6A01.1, d.h. Stottern und Poltern) als Sprechstörung in Ergänzung zur Artikulationsstörung (6A01.0) klassifiziert wird. Zudem wird die Sprachentwicklungsstörung (6A01.2) von der entwicklungsbedingten Artikulations- und Redeflussstörung getrennt.

- Hinsichtlich der Sprachentwicklungsstörung sind in der ICD-11 nun Spezifizierungen möglich. Neben der Sprachentwicklungsstörung mit rezeptiver und expressiver (6A01.20) oder vorwiegend expressiver Beeinträchtigung (6A01.21) sowie anderer unspezifischer Beeinträchtigung (6A01.23) wird zusätzlich analog zum DSM-5 die neue Diagnose der sozialen (pragmatischen) Kommunikationsstörung (6A01.22) als weitere Unterkategorie der Sprachentwicklungsstörung (6A01.2) eingeführt. In der ICD-10 waren diese Spezifizierungen direkt unter der Oberkategorie F80: Umschriebene Entwicklungsstörungen des Sprechens und der Sprache aufgeführt.

- In Analogie zur ICD-10 wird auch in der ICD-11 die Kategorie „Auditive Verarbeitungs- und Wahrnehmungsstörung“" (engl. „Auditory Processing Disorder“) nicht aufgenommen. Diese war als neue ICD-10-Subklassifi- 
Tabelle 2. Klassifikation von Kommunikations-, Sprech- und Sprachstörungen nach ICD-11, ICD-10, DSM-5 und CATALISE-2

\begin{tabular}{|c|c|c|c|c|}
\hline & ICD-11 & ICD-10 & DSM-5 & CATALISE-2 \\
\hline Kapitel & $\begin{array}{l}\text { Neuroentwicklungsstörungen (neu- } \\
\text { rodevelopmental disorders) }\end{array}$ & F8: Entwicklungsstörungen & $\begin{array}{l}\text { Störungen der neuro- } \\
\text { nalen und mentalen } \\
\text { Entwicklung }\end{array}$ & $\begin{array}{l}\text { Sprach-, Sprech- und } \\
\text { Kommunikations- } \\
\text { bedürfnisse (KUSK) }\end{array}$ \\
\hline Oberkategorien & $\begin{array}{l}\text { 6A01: Entwicklungsstörungen des } \\
\text { Sprechens oder der Sprache (ESS) }\end{array}$ & $\begin{array}{l}\text { F80: Umschriebene } \\
\text { Entwicklungsstörungen } \\
\text { des Sprechens und der } \\
\text { Sprache }\end{array}$ & $\begin{array}{l}\text { Kommunikations- } \\
\text { störungen }\end{array}$ & $\begin{array}{l}\text { Sprachstörung und } \\
\text { Sprech-Laut-Störung } \\
\text { (Aussprachestörung) }\end{array}$ \\
\hline Kategorien & $\begin{array}{l}\text { Sprechen } \\
\text { - 6A01.0: Entwicklungsbedingte } \\
\text { Artikulationsstörung } \\
\text { - 6A01.1: Entwicklungsbedingte } \\
\text { Redeflussstörung } \\
\text { Sprache } \\
\text { - 6A01.2: Sprachentwicklungs- } \\
\text { störung } \\
\text { - 6A01.Y: Andere spezifische ent- } \\
\text { wicklungsbedingte Sprech- oder } \\
\text { Sprachstörung } \\
\text { - 6A01.Z: Unspezifische entwick- } \\
\text { lungsbedingte Sprech- oder } \\
\text { Sprachstörung }\end{array}$ & $\begin{array}{l}\text { - F80.0: Artikulations- } \\
\text { störung } \\
\text { - F98.5: Stottern } \\
\text { - F98.6: Poltern } \\
\\
\\
\text { - F80.8: sonstige Entwick- } \\
\text { lungsstörungen des Spre- } \\
\text { chens und der Sprache } \\
\text { - F80.9: nicht näher be- } \\
\text { zeichnete Entwicklungs- } \\
\text { störung des Sprechens } \\
\text { und der Sprache }\end{array}$ & $\begin{array}{l}\text { - 315.39: Artikula- } \\
\text { tionsstörung } \\
\text { - 315.35: Redefluss- } \\
\text { störung mit Beginn in } \\
\text { der Kindheit } \\
\text { - 315.39: Sprach- } \\
\text { störung } \\
\text { - 307.8: Nicht näher } \\
\text { bezeichnete Kom- } \\
\text { munikationsstörung } \\
\text { - 307.9: unspezifische } \\
\text { Kommunikationsstö- } \\
\text { rung }\end{array}$ & $\begin{array}{l}\text { - Artikulationsstörung } \\
\text { - Dysarthrie } \\
\text { - Verbale Dyspraxie } \\
\text { - Redeflussstörung }\end{array}$ \\
\hline $\begin{array}{l}\text { Weitere Unter- } \\
\text { kategorien der } \\
\text { Sprachentwick- } \\
\text { lungsstörung } \\
\text { nach ICD-11: } \\
6 A 01.2\end{array}$ & $\begin{array}{l}\text { - 6A01.20: Sprachentwicklungsstö- } \\
\text { rung mit rezeptiver und expressi- } \\
\text { ver Beeinträchtigung } \\
\text { - 6A01.21: Sprachentwicklungsstö- } \\
\text { rung mit vorwiegend expressiver } \\
\text { Beeinträchtigung } \\
\text { - 6A01.22: Soziale (pragmatische) } \\
\text { Kommunikationsstörung } \\
\text { - 6A01.23: Sprachentwicklungsstö- } \\
\text { rung mit anderer unspezifischer } \\
\text { Beeinträchtigung }\end{array}$ & $\begin{array}{l}\text { - F80.2: rezeptive Sprach- } \\
\text { störung } \\
\text { - F80.1: expressive } \\
\text { Sprachstörung }\end{array}$ & $\begin{array}{l} \\
\text { - 315.39: Soziale } \\
\text { (pragmatische) Kom- } \\
\text { munikationsstörung }\end{array}$ & $\begin{array}{l}\text { - Phonologische } \\
\text { Störung } \\
\text { - Lexikalische Störung } \\
\text { - Grammatische } \\
\text { Störung }\end{array}$ \\
\hline $\begin{array}{l}\text { Sprachbezoge- } \\
\text { ne } \\
\text { Ausschlussdia- } \\
\text { gnosen } \\
\text { (Auswahl) \# }\end{array}$ & $\begin{array}{l}\text { - 6E60.0: Sekundäres Sprech- oder } \\
\text { Sprachsyndrom (SSSS) } \\
\text { - 8A62.2 Erworbene epileptische } \\
\text { Aphasie } \\
\text { - AB50-57: Hörstörungen } \\
\text { - AB5Y: Andere spezifische Erkran- } \\
\text { kungen mit Hörstörungen } \\
\text { - AB5Z: unspezifische Erkrankun- } \\
\text { gen mit Hörstörungen } \\
\text { - AB72: Erkrankungen des akkusti- } \\
\text { schen Nervs } \\
\text { - AB7Y: Andere spezifische Oh- } \\
\text { renerkrankung, die nicht anders- } \\
\text { wo klassifiziert sind } \\
\text { - LD2H Syndromische genetische } \\
\text { Taubheit } \\
\text { - 21: Symptome, Zeichen oder kli- } \\
\text { nische Befunde hinsichtlich der } \\
\text { Sprache oder Stimme (MA 80-82) }\end{array}$ & $\begin{array}{l}\text { - F80.3 Erworbene Aphasie } \\
\text { mit Epilepsie }\end{array}$ & & $\begin{array}{l}\text { - Sprachstörung, die } \\
\text { mit anderen Erkran- } \\
\text { kungen assoziiert ist } \\
\\
\text { - Orofaziale, strukturel- } \\
\text { le Defizite } \\
\text { - Stimmstörung }\end{array}$ \\
\hline
\end{tabular}

Anmerkung: \# Diese Diagnosen schließen die Diagnose einer Sprachentwicklungsstörung aus, gehen jedoch mit Sprach- oder Sprechstörungen einher. 
kation F80.20 hinsichtlich der Spezifizierung einer rezeptiven Sprachstörung im Jahr 2000 vonseiten der Deutschen Gesellschaft für Pädaudiologie und Phoniatrie e.V. für Deutschland vorgeschlagen worden ( $\mathrm{Ni}$ ckisch et al., 2000) und ist in der (abgelaufenen) AWMF-S2k-Leitlinie 049-006 zu Sprachentwicklungsstörungen unter Berücksichtigung umschriebener Sprachentwicklungsstörungen, Diagnostik, sowie in der AWMF-S1-Leitlinie 049-012 Auditive Verarbeitungsund Wahrnehmungsstörungen als nur in Deutschland verbreitete Diagnose eingeführt worden.

- Durch die Trennung der „primären“ Entwicklungsstörungen des Sprechens oder der Sprache (ESS) von 6E60.0: Sekundäres Sprech- oder Sprachsyndrom (SSSS) sowie der in der ICD-11 gewünschten Differenzierung einer organischen Ursache möglicher Sprachund Sprechprobleme wurde die Diagnose der „Erworbenen epileptischen Aphasie" logisch dem Kapitel „Erkrankungen des Nervensystems“ zugeordnet und unter die Epilepsien aufgenommen.

- Dieses Konzept kann auch auf Kinder mit Taubheit übertragen werden: Wenn ein Kind funktional und altersgemäß Gebärdensprache beherrscht, wird nur die Taubheit klassifiziert, aber nicht eine ESS oder SSSS.

Hinsichtlich des Detailvergleichs der diagnostischen Kriterien der Sprachstörungen in ICD-11 und ICD-10 ergeben sich folgende Übereinstimmungen und Unterschiede:

- Sowohl hinsichtlich der rezeptiven und expressiven Sprachstörung als auch hinsichtlich der alleinigen expressiven Sprachstörung wird eine Diskrepanz zu Alter und nonverbalen kognitiven Fertigkeiten (nonverbaler Intelligenzquotient [IQ]) gefordert, der nach den Forschungskriterien der ICD-10 hinsichtlich des Alters zwei Standardabweichungen (SD), hinsichtlich des nonverbalen IQs bei 1 SD liegen muss (Remschmidt, Schmidt \& Poustka, 2017). In der ICD-11 wird ein solches „SD-Kriterium" derzeit nicht explizit definiert, jedoch betont, dass die sprachlichen Fertigkeiten außerhalb der normalen Variabilität eingeschränkt sein müssen (Tabelle 1).

- In der ICD-11 ist eine Intelligenzminderung (IQ < 70) im Gegensatz zur ICD-10 kein Ausschlusskriterium mehr für eine ESS.

- Analog sind in beiden Klassifikationssystemen die psychiatrischen Diagnosen einer Autismus-Spektrum-Störung sowie eines selektiven Mutismus Ausschlusskriterien, ebenso organische Grunderkrankungen des Nervensystems oder eine Taubheit. Eine Hörstörung ist in beiden Versionen nicht als Ausschlusskriterium genannt, allerdings entspricht der ICD-11-Logik implizit, dass bei Sprach- und Sprechproblemen einhergehend mit einer Hörstörung zunächst geprüft werden muss, ob diese ausschließlich durch die Hörstörung bedingt sind, dann wäre ein SSSS (6E60.0) als Diagnose zu vergeben. Falls nach erfolgreicher und abgeschlossener Therapie der zugrunde liegenden Erkrankung (wie z.B. Mittelohrerguss) weiterhin Sprach- oder Sprechprobleme vorhanden sind, kann nach erfolgreicher Behandlung der Hörstörung die Diagnose einer Unterkategorie der Entwicklungsstörungen des Sprechens oder der Sprache (6A01) erfolgen.

- Analog ist in beiden Klassifikationssystemen erlaubt, weitere psychiatrische Diagnosen mit Ausnahme einer Autismus-Spektrum-Störung oder eines selektiven $\mathrm{Mu}$ tismus zusätzlich zu der Diagnose einer Sprech- oder Sprachstörung zu vergeben.

- Über ICD-10 hinaus ist die soziale (pragmatische) Kommunikationsstörung analog zu DSM-5 neu in die ICD-11 aufgenommen worden. Sie ist charakterisiert durch eine primäre Einschränkung der Pragmatik, d.h. einer dauerhaften und deutlichen Einschränkung, Sprache im sozialen Kontext zu verstehen und zu benutzen (z.B. Anpassung der Sprache an implizite Gesprächsregeln, Erkennen und Korrigieren von Missverständnissen, Umgang mit sprachlichen Zweideutigkeiten und nichtwörtlichen Ausdrücken). Die Entwicklungs-, Diskrepanz- und weiteren Ausschlusskriterien, auch hinsichtlich Autismus-Spektrum-Störung und selektivem Mutismus, entsprechen den anderen Sprachentwicklungsstörungen.

Hinsichtlich des Detailvergleichs der diagnostischen Kriterien der Sprechstörungen in ICD-11 und ICD-10 ergeben sich ergänzend folgende Unterschiede:

- Vergleichbar zu den Sprachstörungen wird das AltersDiskrepanzkriterium in der ICD-11 für die Artikulations- und die Redeflussstörung zwar gefordert, aber nicht spezifiziert (ICD-10:2 SD).

- Nur für die Artikulationsstörung wird auch ein Diskrepanzkriterium zum nonverbalen IQ erwähnt (ICD-10: 1 SD, ICD-11: nicht spezifiziert).

- In der ICD-11 ist eine Intelligenzminderung (IQ< $<0) \mathrm{im}$ Gegensatz zur ICD-10 kein Ausschlusskriterium mehr für eine Artikulationsstörung; hinsichtlich des Stotterns und Polterns war dieses Ausschlusskriterium auch in der ICD-10 nicht gegeben.

- Hinsichtlich der entwicklungsbedingten Artikulationsstörung wird in der ICD-11 spezifisch die Dysarthrie (MA80.2) abgegrenzt, die jedoch derzeit noch nicht genau definiert ist. In der ICD-10 erfolgte eine Abgrenzung der Artikulationsstörung von einer Apraxie.

Allgemeine Kriterien für Sprech- oder Sprachstörungen, dass diese früh in der Entwicklung auftreten und über längere Zeit persistieren sowie zu deutlichen Einschränkungen in der Kommunikation und Verständlichkeit führen müssen, sind in beiden ICD-Versionen vergleichbar; eben- 
so das Kriterium, dass die Sprech- oder Sprachstörung nicht durch soziale, kulturelle oder andere Umweltvariablen (wie z.B. regionaler Dialekt) sowie eine neurologische Erkrankung oder eine Hörstörung (s.o.) erklärt werden kann.

\section{Kritische Bewertung}

Aus dem oben Dargestellten ergibt sich, dass die ICD-11 hinsichtlich der Klassifikation in einer Kontinuität mit ICD-10 und DSM-5 steht. Einige vorgenommene Änderungen sind hinsichtlich des aktuellen Forschungsstandes zu Sprach- und Sprechstörungen sowie hinsichtlich des klinischen Gebrauchs weiterführend, wie z.B. die Differenzierung in die (primäre) ESS oder das SSSS bei neurologischer, sensorischer oder anderer somatischer Grunderkrankung sowie die Trennung von Sprach- und Sprechstörungen. In Bezug auf letztere Differenzierung wäre es allerdings logisch gewesen, neben der Kategorie Sprachstörung (mit Spezifizierungen) eine Kategorie Sprechstörung mit den beiden Spezifizierungen (= Unterkategorien) der Artikulations- und der Redeflussstörung vorzunehmen.

Erfreulich ist, dass Sprach- und Sprechstörungen sich nicht mehr gegenseitig ausschließen, sondern als komorbide Erkrankungen diagnostiziert werden können; ebenso sind auch die meisten psychischen Störungen als komorbide Diagnosen erlaubt. Die - analog zur ICD-10 - als Ausschlussdiagnosen einer Sprachstörung aufgeführten Diagnosen Autismus-Spektrum-Störung und selektiver Mutismus sind allerdings aufgrund des aktuellen Forschungsstandes nicht nachvollziehbar. Es gibt eine Subgruppe von Kindern und Jugendlichen mit diesen Störungsbildern, die eine über die Diagnosekriterien von Autismus-Spektrum-Störung oder selektivem Mutismus hinausgehende zusätzliche Sprachstörung oder eben auch keine Sprachstörung aufweisen. In der CCC-R, einer Skala, die pragmatische und grammatische Sprachprobleme erfasst, zeigte sich z.B. kein Unterschied in der Ausprägung sowie der Varianz der grammatischen Sprachprobleme (die einer expressiven Sprachstörung entsprechen) zwischen Kindern und Jugendlichen mit Autismus-Spektrum-Störung oder solchen mit anderen psychischen Störungen. Hinsichtlich der pragmatischen Fertigkeiten zeigten sich hingegen deutlich stärkere Einschränkungen bei Autismus-Spektrum-Störung als bei Kindern und Jugendlichen mit anderen psychischen Störungen (Wellnitz et al., 2021). Daher erscheint es gerechtfertigt, die Diagnosen 6A01.20 (Sprachentwicklungsstörung mit rezeptiver und expressiver Beeinträchtigung) und 6A01.21 (Sprachentwicklungsstörung mit vorwie- gend expressiver Beeinträchtigung) entgegen ICD-10 und ICD-11 bei Autismus-Spektrum-Störung als komorbide Erkrankungen zu erlauben, die Diagnose 6A01.22 (Soziale [pragmatische] Kommunikationsstörung) jedoch als Differenzialdiagnose zur Autismus-SpektrumStörung abzugrenzen. Ebenso gibt es auch bei Kindern mit selektivem Mutismus eine Gruppe, die zusätzliche Sprachstörungen aufweist, die nicht alleine durch den selektiven Mutismus bzw. der selektive Mutismus nicht ausschließlich durch die Sprachstörung erklärbar ist (Cohan et al., 2008). Es wäre deshalb zu empfehlen, sowohl eine Autismus-Spektrum-Störung als auch einen selektiven Mutismus als Komorbidität zumindest von 6A01.20 und 6A01.21 zu erlauben. Die diagnostische Validität der $\approx$ sozialen (pragmatischen) Kommunikationsstörung (Norbury, 2014) sowie die Abgrenzung zur AutismusSpektrum-Störung (Freitag, 2020) ist aktuell weiterhin Gegenstand der Forschung; hier können noch keine weiterführenden Aussagen getroffen werden.

Sehr begrüßenswert ist die Klassifikation der Redeflussstörungen unter die Sprechstörungen in der ICD-11 und nicht mehr bei „Andere Verhaltens- und emotionale Störungen mit Beginn in der Kindheit und Jugend". Dies entspricht der Erkenntnis, dass Sprechstörungen Entwicklungsstörungen und nicht primär emotionale oder Verhaltensstörungen sind, auch wenn Letztere als komorbide Diagnosen vorkommen können.

Eine weitere erfreuliche Neuerung in der ICD-11 ist die differenzialdiagnostische Differenzierungs- und Abgrenzungsmöglichkeit von 6A01 ESS und 6E60.0 SSSS. Dies bedeutet, dass alle mit einer neurologischen, motorischen und Hörstörungen ausschließlich einhergehenden Sprach- und Sprechprobleme nicht mehr als 6A01 ESS klassifiziert werden dürfen, sondern die Diagnose einer 6E60.0 SSSS oder ggf. auch einer anderen spezifischen neurologischen, motorischen, sensorischen oder somatischen Erkrankung direkt zu geordnet werden müssen. Diese Differenzierung ist insbesondere hinsichtlich der notwendigen interdisziplinären diagnostischen Kompetenz sowie der Behandlung der organischen Grunderkrankung wichtig und sinnvoll. Allerdings wird es in der Praxis teilweise schwierig werden, $\mathrm{zu}$ entscheiden, ob eine 6A01 ESS oder eine 6E60.0 SSSS vorliegt. Als Beispiel seien hier genetische Diagnosen genannt. Sehr viele Sprach- und Sprechstörungen sind durch genetische Risikofaktoren entweder direkt verursacht oder zumindest in ihrer Ausprägung beeinflusst (Hildebrand et al., 2020; Verhoef, Shapland, Fisher, Dale \& St Pourcain, 2021). Bei fortschreitender Forschung werden weitere genetische Erkrankungen beschrieben werden, die mit Sprach- und Sprechproblemen einhergehen. Ein anderes Beispiel wäre das Vorliegen eines Paukenergusses, der zu einer Hörstörung geführt hat. Solange die Hörstörung anhand 
eines Hörtests noch nachweisbar ist und gleichzeitig eine Sprech- oder Sprachstörung vorliegt, sollte hier eine 6E60.0 SSSS diagnostiziert werden. Wenn nach Sanierung des Paukenergusses und unauffälligem Hörtest weiterhin eine Sprech- oder Sprachstörung vorliegt, so ist von einer 6A01 ESS auszugehen und die Diagnose wäre entsprechend zu ändern und zu spezifizieren.

Um diese teilweise schwierige Differenzierung gut und transparent beschreiben zu können, wird im nächsten $\mathrm{Ab}$ schnitt (Klinischer Ausblick) ein Vorschlag gemacht, wie die Klassifikation von in der frühen kindlichen Entwicklung vorliegenden Sprach- und Sprechproblemen entsprechend des Multiaxialen Klassifikationsschemas vorgenommen werden kann, um diese Klassifikationsschwierigkeiten zulösen.

Die wesentlichen Einschränkungen der ICD-11 sind in der nicht ausreichenden, weder linguistisch noch entwicklungspsychologisch basierten Subdifferenzierung der 6A01 ESS zu finden. Allerdings können diese Spezifi$\mathrm{ka}$ in die ICD-11-Klassifikation sehr gut integriert werden (ein Vorschlag findet sich anbei in den Tabellen 3 und 4).

Da im englischen Sprachraum aktuell der Versuch unternommen wird, eine Konsensus-Klassifikation von Sprachproblemen im Kindesalter insbesondere vonseiten unterschiedlicher Sprachtherapeut_innen zu etablieren, die auch im deutschsprachigen Raum (Kauschke \& Vogt, 2019) sowie hinsichtlich der Überarbeitung der AWMFS2k-Leitlinie 049-006 diskutiert wird, soll dieser „CATALISE-2“-Vorschlag hier auch erwähnt und in Relation zur ICD-11 gesetzt und bewertet werden. In dem der CATALISE-2-Klassifikation zugrunde liegenden DELPHIProzess war zunächst das Ziel, Kriterien zu entwickeln, die es erleichtern können, Kinder zu identifizieren, die von Sprach- und Sprechtherapie profitieren können. In der ersten Publikation (Bishop, Snowling, Thompson \& Greenhalgh, 2016) wird explizit darauf hingewiesen, dass ICD-10- und DSM-5-Kriterien von vielen Sprachtherapeut_innen nicht beachtet oder als unzureichend eingeschätzt werden. Im zweiten Artikel (Bishop, Snowling, Thompson \& Greenhalgh, 2017) wird eine neue Terminologie für die Klassifikation (CATALISE-2) vorgeschlagen (siehe Tabelle 2). Bei Kindern mit Sprachentwicklungsproblemen soll - nach Ausschluss von sprachlichen Problemen im Rahmen des Zweitspracherwerbs bei unauffälliger muttersprachlicher Entwicklung - demnach nur die Unterscheidung getroffen werden, ob eine Sprachentwicklungsstörung mit einer somatischen einschließlich genetischen Grunderkrankung, Autismus-Spektrum-Störung oder Intelligenzminderung einhergeht oder nicht. Das Diskrepanzkriterium zum nonverbalen IQ, das in der ICD-11 gefordert wird, wird explizit in dieser Klassifikation abgelehnt. Artikulations- und Redeflussstörungen werden als mögliche komorbide Störungen der Sprachentwicklungsstörung (ohne somatische Ursache) erwähnt. Ebenso dürfen andere psychische Störungen, wie die Aufmerksamkeitsdefizit- und Hyperaktivitätsstörung (ADHS), emotionale Störungen oder auch Dyslexie als komorbide Störungen einer genuinen Sprachentwicklungsstörung klassifiziert werden. Hinsichtlich spezifischer Subgruppen innerhalb der Sprachentwicklungsstörung konnte die Gruppe keinen Konsens erreichen. Es wird allerdings darauf hingewiesen, dass es Kinder gibt, die unterschiedliche sprachliche Einschränkungen in den folgenden Bereichen aufweisen können: Phonologie,

Tabelle 3. Aufeinander aufbauende Sprachkomponenten im Rahmen der Sprachentwicklung

\begin{tabular}{|c|c|c|}
\hline Sprachkomponente & Aspekte & Vorläuferfähigkeiten \\
\hline Phonetik & Artikulation & $\begin{array}{l}\text { Auditorische Perzeption und Aufmerksamkeit, orale } \\
\text { Motorik, Imitation }\end{array}$ \\
\hline Phonologie & $\begin{array}{l}\text { Lautwahrnehmung, Lautproduktion, Prosodie, phonolo- } \\
\text { gische Prozesse }\end{array}$ & $\begin{array}{l}\text { Auditorische Perzeption und Aufmerksamkeit, orale } \\
\text { Motorik, Imitation }\end{array}$ \\
\hline $\begin{array}{l}\text { Lexikon (lexikalische } \\
\text { Semantik) }\end{array}$ & $\begin{array}{l}\text { Lexikalische Erwerbsmechanismen, aktiver und passi- } \\
\text { ver Wortschatz, Komposition des Lexikons }\end{array}$ & Phonologie, Konzeptbildung \\
\hline Morpho-Syntax & $\begin{array}{l}\text { Satzbau, Flexion der Verben, Kasus und Singular/Plural } \\
\text { bei Nomen }\end{array}$ & Phonologie, Konzeptbildung, Lexikon \\
\hline Satzsemantik & $\begin{array}{l}\text { Verständnis von w-Fragen, Mengenausdrücken (jeder, } \\
\text { alle), Pronomen, Negation }\end{array}$ & $\begin{array}{l}\text { Phonologie, Morpho-Syntax, Gedächtnis, Wissens- } \\
\text { repräsentation, Kategorisierung, Konzeptbildung, Lern- } \\
\text { und Denkprozesse, soziale Interaktion }\end{array}$ \\
\hline Pragmatik & Sprachhandlungen, Ironie, Argumentationsausdrücke & $\begin{array}{l}\text { Syntax, Lexikon, Satzsemantik, nonverbale } \\
\text { Kommunikation, soziale Perzeption, soziale Motivation } \\
\text { und soziale Kognition }\end{array}$ \\
\hline
\end{tabular}


Tabelle 4. Therapieorientierter Klassifikationsvorschlag nach ICD-11 und dem Multiaxialen Klassifikationsschema

\begin{tabular}{|c|c|c|}
\hline $\begin{array}{l}\text { Multiaxiale Klassifikation } \\
\text { (Remschmidt et al., 2017) }\end{array}$ & ICD-11 & Linguistische Feindifferenzierung \\
\hline $\begin{array}{l}\text { Achse I: Psychische Stö- } \\
\text { rung }\end{array}$ & $\begin{array}{l}\text { Gesamtes Kapitel } 06 \text { (außer 6A00, 6A01, 6A03, 6A04) } \\
\text { möglich: alle komorbiden psychischen Störungen er- } \\
\text { laubt }\end{array}$ & \\
\hline $\begin{array}{l}\text { Achse II: Teilleistungsstö- } \\
\text { rung (ICD-11:6A01, 6A03, } \\
6 A 04)\end{array}$ & $\begin{array}{l}\text { Sprechen } \\
\text { - 6A01.0: Entwicklungsbedingte Artikulationsstörung } \\
\text { - 6A01.1: Entwicklungsbedingte Redeflussstörung } \\
\text { Sprache } \\
\text { - 6A01.20: Sprachentwicklungsstörung mit rezeptiver } \\
\text { und expressiver Beeinträchtigung } \\
\text { - 6A01.21: Sprachentwicklungsstörung mit vorwiegend } \\
\text { expressiver Beeinträchtigung } \\
\text { - 6A01.22: Soziale (pragmatische) Kommunikations- } \\
\text { störung }\end{array}$ & $\begin{array}{l}\text { - Artikulationsstörung; ggf. Spezifizierung der nicht } \\
\text { korrekt ausgesprochenen Konsonanten } \\
\text { - Redeflussstörung; ggf. Spezifizierung von patho- } \\
\text { logischer Atemtechnik und Einschränkungen der } \\
\text { Sprachmotorik und Koordination } \\
\text { - Einschränkung der phonologischen Komponente } \\
\text { - Einschränkungen der morphologisch-syntakti- } \\
\text { schen Ebene } \\
\text { - Einschränkung der lexikalisch-semantischen } \\
\text { Komponente } \\
\text { - Einschränkungen der satzsemantischen Ebene } \\
\text { - Einschränkungen der pragmatischen Ebene }\end{array}$ \\
\hline $\begin{array}{l}\text { Achse III: Kognitive Fertig- } \\
\text { keiten (ICD-11:6A00) }\end{array}$ & $\begin{array}{l}\text { Nonverbaler IQ } 1 \text { bis } 1.5 \text { SD über standardisiert erfass- } \\
\text { ten sprachlichen Fertigkeiten }\end{array}$ & \\
\hline $\begin{array}{l}\text { Achse IV: Somatische } \\
\text { Störung }\end{array}$ & $\begin{array}{l}\text { Internistisch-neurologische Erkrankung kann kodiert } \\
\text { werden, darf aber Symptomatik der Entwicklungsstö- } \\
\text { rung der Sprache und des Sprechens nicht ausschließ- } \\
\text { lich verursachen. } \\
\text { Genetische Befunde, die Sprech- und Sprachstörung } \\
\text { erklären, können hier ebenfalls aufgeführt werden. }\end{array}$ & \\
\hline $\begin{array}{l}\text { Achse V: Psychosoziale } \\
\text { Umstände }\end{array}$ & $\begin{array}{l}\text { Können kodiert werden, dürfen aber Symptomatik der } \\
\text { Entwicklungsstörung der Sprache und des Sprechens } \\
\text { nicht ausschließlich begründen }\end{array}$ & \\
\hline $\begin{array}{l}\text { Achse VI: Globales } \\
\text { Funktionsniveau }\end{array}$ & Integrierte Betrachtung über Achse I bis Achse V hinweg & \\
\hline
\end{tabular}

Syntax, Wortfindung und Semantik, Pragmatik, verbales Lernen und Gedächtnis.

Es ist aus der Darstellung deutlich geworden, dass die CATALISE-2-Klassifikation nicht dem aktuellen Forschungsstand entspricht. Sowohl die entwicklungspsychologische und linguistische Forschung zur Sprachentwicklung als auch die somatische (Pädaudiologie/HNO, Neurologie) und kinderpsychiatrische Forschung können zahlreiche neue Erkenntnisse beitragen, die in der CATALISE-2-Klassifikation nicht aufgenommen wurden, da diese Expertise in dem „Expertenpannel“ nicht vertreten war. Die Differenzierung zwischen Sprachentwicklungsstörung ohne bisher definierte Ursache und Sprachentwicklungsstörung, die mit anderen Erkrankungen assoziiert ist, ist sehr unspezifisch und führt weder hinsichtlich der notwendigen Interventionsplanung noch hinsichtlich der Forschung weiter. Wie oben schon diskutiert, ist davon auszugehen, dass Sprach- und Sprechstörungen zumindest in Ländern mit einem öffentlichen Gesundheitssystem, guter Schwangerschaftsvorsorge und guter perinataler Versorgung überwiegend genetisch bedingte Ursachen haben, einerseits im Sinne einer Vulnerabilität bei polygenetischem Risiko, andererseits im Sinne einer direkten Kausalität bei hoch penetranten monogenen Erkrankungen oder Mikrodeletions- oder -duplikationssyndromen (Barnett \& van Bon, 2015; Hildebrand et al., 2020). Hier kann es selbstverständlich im Sinne der gut belegten Pleiotropie zahlreicher genetischer Risikofaktoren Überlappungen mit Risikogenen für Autismus-Spektrum-Störung, ADHS, Epilepsie, Dyslexie oder Intelligenzminderung geben. Zudem finden sich, wie in der ICD-11 gut durch die Diagnose 6E60.0 SSSS abgebildet, Sprach- und Sprechprobleme bei den unterschiedlichsten Formen von Erkrankungen, die mit Höreinschränkungen einhergehen, bei neurologischen (einschließlich motorischen) Erkrankungen. Auch das Fehlen des Diskrepanzkriteriums hinsichtlich normaler Alters- und Sprachentwicklungsvariabilität ist ein weiteres deutliches Zeichen einer fehlenden diagnostischen Validität der CATALISE-2-Klassifikation. 
Allen derzeit verfügbaren Klassifikationen ist zudem gemeinsam, dass zentrale Befunde der jüngsten internationalen Spracherwerbsforschung bislang nicht hinreichend Eingang in die Zuordnung zu den jeweiligen Untergruppen gefunden haben. Hier sei insbesondere auf die folgenden drei Aspekte hingewiesen, die wichtige Folgen für die Erkennung von Sprachstörungen, die Diagnosestellung sowie für die zu verordnende Therapie haben: Störungen auf der Ebene der Satzsemantik, selektive Störungen einzelner sprachlicher Ebenen und der frühe Zweitspracherwerb.

\section{Satzsemantik}

Zwar werden semantische Störungen als Kriterium einer ESS in zahlreichen Klassifikationen genannt, jedoch sind hiermit typischerweise Probleme auf lexikalischer Ebene, also hinsichtlich des Wortschatzes, gemeint (Kauschke \& Vogt, 2019). Lexikalisch-semantische Störungen äußern sich in der Produktion durch einen geringeren Wortschatzumfang oder durch schwächere Leistungen im Wortabruf; zudem finden sich u. a. Belege für Verstehensprobleme mit bestimmten Verben. Probleme mit der Bedeutung von Sätzen, also der Satzsemantik, äußern sich u. a. durch ein inkorrektes Verständnis von bestimmten W-Fragen sowie Sätzen mit Mengenausdrücken und mit Pronomen (Schulz \& Grimm, 2012). Defizite auf der Ebene der Satzsemantik äußern sich in erster Linie rezeptiv und sind daher ohne systematische Tests schwer erkennbar; sie haben jedoch erhebliche Auswirkungen auf das Sprachverständnis der Kinder, in Gesprächen und beim Lesen von Texten.

\section{Selektive Störungen}

Das heterogene Störungsbild der Sprachstörungen folgt nicht nur daraus, dass die Störung prinzipiell alle Bereiche des Sprachsystems betreffen kann, sondern auch daraus, dass Teilbereiche jeweils selektiv gestört sein können. So sind manche Kinder von einer ESS selektiv im Bereich der Syntax betroffen, andere im Bereich der Phonologie oder der Pragmatik. Die bestehenden Klassifikationen bilden diese Möglichkeit einer selektiven Störung - mit Ausnahme der pragmatischen Störung - nicht ab, sodass die Gefahr besteht, dass Kinder, die in ihrer Sprachproduktion weder phonologisch noch grammatisch, also morpho-syntaktisch, auffällig sind, nicht weiter untersucht werden. Aus diesem Grund wird als Zusatzspezifizierung einer ICD-11-Diagnose in Tabelle 4 vorgeschlagen, jeweils die sprachlichen Bereiche, die eingeschränkt sind und deshalb besonders gefördert werden müssen, bei einer Diagnosestellung aufzuführen.

\section{Mehrsprachigkeit und Sprachstörung}

Mehrsprachige Kinder sind ebenso häufig wie einsprachige Kinder von einer ESS betroffen. Die Aufgabe, Kinder mit Deutsch als Zweitsprache (DaZ) mit einer ESS von typischen Zweitsprachlerner_innen zu unterscheiden, ist jedoch wegen der Heterogenität innerhalb der Gruppe der Mehrsprachigen aufgrund von Unterschieden hinsichtlich der Erwerbsbiografie wesentlich komplexer als für einsprachige Kinder. In Anbetracht des kürzeren Kontakts mit dem Deutschen und des höheren Alters bei Spracherwerbsbeginn lassen sich die Sprachfähigkeiten von Kindern mit DaZ nicht einfach mit denen monolingualer Gleichaltriger vergleichen. Bewertet man ein- und mehrsprachige Kinder nach denselben Maßstäben, schneiden frühe Zweitsprachlerner_innen in der Regel unterdurchschnittlich ab, weil sie die Zweitsprache Deutsch zeitlich versetzt zur Erstsprache erwerben, obwohl sie sich gemessen an ihrer (naturgemäß kürzeren) Kontaktdauer zur deutschen Sprache durchaus adäquat entwickelt haben. Gleichzeitig zeigen viele internationale Befunde, dass sich anhand der Äußerungen mehrsprachiger Kinder nicht zweifelsfrei entscheiden lässt, ob sie eine ESS aufweisen oder nicht (Grimm \& Schulz, 2014). Daher ist einerseits das Ausschlusskriterium der ICD-11 hinsichtlich Zweisprachigkeit etc. (Tabelle 1) zu vage; andererseits spiegelt der in der deutschsprachigen Diskussion von CATALISE-2 vorgeschlagene Begriff „Umgebungsbedingte Sprachauffälligkeiten“" (Kauschke \& Vogt, 2019) das Dilemma wider, diesen Kindern Unterstützungsbedarf in den Bereichen Sprache und Kommunikation zuzugestehen, ohne den Zweitspracherwerb zu pathologisieren. Will man an der Trennung von Sprachtherapie (die sich an Kinder mit ESS etc. wendet, von ärztlichem Fachpersonal verordnet und von Sprachtherapeut_innen etc. durchgeführt wird) und Sprachförderung (für sich sprachlich typisch entwickelnde Kinder, initiiert durch Kita oder Schule und durchgeführt von pädagogischen Fachkräften) festhalten, ist die Berücksichtigung der DaZ-Kinder mit Förderbedarf als „KUSK“ (Kinder mit Unterstützungsbedarf in den Bereichen Sprache/Kommunikation) in CATALISE-2 kritisch zu sehen. Da die Erforschung des gestörten kindlichen Zweitspracherwerbs unter Migrationsbedingungen erst in den letzten rund 10 Jahren intensiviert wurde (siehe www.bisli.org), überrascht der Mangel an geeigneten diagnostischen Verfahren nicht. In der Spracherwerbsforschung wird argumentiert, dass nur separate DaZ-Normen es erlauben, zuverlässig festzustellen, ob ein DaZ-Kind aufgrund einer ESS eine Therapie benötigt oder ob lediglich Förderbedarf in der Zweitsprache besteht, der aus der kürzeren Erwerbszeit resultiert, mit der Erwartung, dass die Kinder rasch Fortschritte machen (Schulz, 2013; Schulz \& Tracy, 2011; Voet Cornelli, Schulz \& Tracy, 2013). 


\section{Klinischer Ausblick}

Wie ist nun vorzugehen, wenn bei einem Kind der Verdacht auf eine Sprech- oder Sprachstörung vorliegt?

Zunächst muss der Entwicklungsstand der Sprache und des Sprechens möglichst objektiv beschrieben werden. Hinsichtlich der Sprachentwicklung gibt es deutschsprachige, normierte Tests für das Kleinkind- und Vorschulalter, wie z. B. den umfassenden Entwicklungstest Bayley-III (Reuner \& Rosenkranz, 2014) oder die Sprachentwicklungstests SETK2 und SETK3-5 (Grimm, 2015, 2016). Wie oben ausgeführt, sind diese an einsprachigen Kindern normierten Tests für mehrsprachige Kinder jedoch wenig geeignet, da die passende Vergleichsnorm fehlt. Der Sprachtest LiSe-DaZ (Schulz \& Tracy, 2011) verfügt über separate Nomen für einsprachige Kinder und für DaZ-Kinder, ist allerdings für Kinder mit Autismus-Spektrum-Störung aufgrund der für die Testung notwendigen nonverbalen Kommunikations- und symbolischen Fertigkeiten nur bedingt geeignet. Parallel dazu muss das nonverbale Entwicklungsalter anhand entsprechender Entwicklungsoder Intelligenztests bestimmt werden. Darauf basierend kann die Diskrepanz zwischen Sprachentwicklung und Alter bzw. nonverbaler kognitiver Entwicklung bestimmt werden. Zur Diagnostik von Sprechstörungen liegt aktuell leider kein entsprechend normiertes Untersuchungsinstrument vor. Hinsichtlich des Diskrepanzkriteriums bietet sich an, unabhängig von der nonverbalen Intelligenz dieselben Kriterien zu verwenden, wie sie in den AWMF-S3Leitlinien 028-044 Diagnostik und Behandlung bei der Lese- und/oder Rechtschreibstörung oder 028-046 Diagnostik und Behandlung der Rechenstörung vorgeschlagen werden (je nach klinischer Konstellation 1 oder 1.5 SD; siehe AWMF.org).

Zudem muss parallel (oder bei entsprechenden wegweisenden Symptomen auch als erster Schritt) eine gründliche internistisch-neurologische sowie pädaudiologische Untersuchung erfolgen, um eine behandelbare somatische Erkrankung auszuschließen. Sollten Dysmorphiezeichen, eine Intelligenzminderung, Hörstörungen und andere hinsichtlich einer genetischen Grunderkrankung wegweisende Symptome vorliegen, sollte auch eine humangenetische Abklärung erfolgen. International wird sogar - analog zum Vorgehen bei Autismus-Spektrum-Störung oder Intelligenzminderung - generell eine humangenetische Abklärung empfohlen, wenn eine ESS vorliegt (Barnett \& van Bon, 2015). Wenn eine somatische Erkrankung vorliegt, die zu behandlungsbedürftigen Sprach- oder Sprechauffälligkeiten geführt hat, muss ein SSSS (6E60.0) und darf keine ESS (6A01) diagnostiziert werden.

Hinsichtlich der nur in Deutschland von Pädaudiolog_ innen verwendeten Diagnose „Auditive Verarbeitungsund Wahrnehmungsstörung" (Nickisch et al., 2000) ist zu betonen, dass diese keine somatische, neurologische, motorische oder sensorische Erkrankung im Sinne der ICD-11 darstellt, da die Phonologie ein genuiner Teil des Sprachsystems ist (siehe Tabelle 3). Somit muss eine Einschränkung der Phonologie, die auf einer entsprechenden neuronalen Verarbeitungsstörung beruht, als genuiner Teil der Diagnose 6A01.20: Sprachentwicklungsstörung mit rezeptiver und expressiver Beeinträchtigung interpretiert und entsprechend klassifiziert werden. In Tabelle 4 wird vorgeschlagen, dass hinsichtlich der linguistisch nicht ausreichend definierten ICD-11-Diagnosen 6A01.20 und 6A01.21 zusätzlich eine linguistische Feindifferenzierung vorgenommen werden kann, um hier für die Therapie wesentlichen Aspekte mit zu erfassen.

Des Weiteren sollten in jedem Fall orientierend mögliche komorbide psychische Störungen abgeklärt werden, da bei Kindern mit Sprach- und Sprechstörungen gehäuft zahlreiche psychische Störungen als komorbide Erkrankungen vorkommen, die ebenfalls so früh wie möglich therapiert werden sollten, um eine Chronifizierung zu vermeiden.

Gemäß der Multiaxialen Klassifikation psychischer Störungen (MAS; Remschmidt et al., 2017) können psychische und somatische Erkrankungen parallel zu Teilleistungsstörungen diagnostiziert werden, wenn diese nicht die Sprach- und Sprechprobleme des Kindes ausreichend erklären. In Tabelle 4 ist dazu ein entsprechender Vorschlag zu finden. Die ICD-11 selbst kennt den Begriff einer "Teilleistungsstörung" nicht, nennt aber das doppelte Diskrepanzkriterium zu Alter und nonverbalem IQ bei den Entwicklungsstörungen des Sprechens oder der Sprache (6A01), bei den Lernentwicklungsstörungen (,developmental learning disorder“, 6A03), die im Wesentlichen spezifische Störungen des Lesens, Schreibens und Rechnens abbilden, und der motorischen Koordinationsstörung („,developmental motor coordination disorder“, 6A04). Insofern sind bei diesen Diagnosen implizit die Kriterien einer Teilleistungsstörung gegeben.

Über die ICD-11 hinausgehend schlagen wir - wie oben begründet - vor, auf der ersten Achse des MAS auch die Diagnose einer Autismus-Spektrum-Störung oder eines selektiven Mutismus zu erlauben, wenn diese Diagnosen die sprachlichen Auffälligkeiten des Kindes nicht vollständig erklären, da dies wesentliche Implikationen für eine effektive Therapie der Sprach- oder Sprechstörung des Kindes nach sich zieht. Ebenso sollte die 6A01 (Entwicklungsstörung) klassifiziert werden können, auch wenn ein genetischer Befund diese erklären kann. Die Diagnose 6E60.0 (Sekundäres Sprach- oder Sprechsyndrom) sollte immer dann bei genetischen Grunderkrankungen diagnostiziert werden, wenn diese primär zu Hör- oder neurologischen Störungen führen, die ihrerseits die Sprachprobleme des Kindes verursachen. Dies entspricht dem Stand 
der humangenetischen Forschung, die gezeigt hat, dass die meisten Sprachentwicklungsstörungen sowie die Redeflussstörung überwiegend eine genetische Ätiologie aufweisen.

Die Möglichkeit, statt einer ESS ein SSSS zu diagnostizieren, erlaubt es, sprachliche Förderung bei entsprechender Notwendigkeit auch Kindern zukommen zu lassen, die nicht die Diagnosekriterien einer ESS erfüllen.

\section{Forschungsausblick}

Aus der obigen Darstellung ist unmittelbar zu entnehmen, dass es für eine effektive Forschung hinsichtlich Sprachund Sprechstörungen zunächst einer breit abgestimmten Klassifikation bedarf, die über alle Disziplinen zum einen akzeptiert und zum anderen angewendet werden sollte. Die Forschung zu Sprach- und Sprechstörungen ist aufgrund der Komplexität der menschlichen Sprache immer interdisziplinär und muss sowohl die Entwicklungspsychologie, die Linguistik, die Logopädie, die Humangenetik, die Pädaudiologie als auch die Kinder- und Jugendpsychiatrie und -psychotherapie umfassen, um einerseits die umfassende Diagnostik einschließlich möglicher komorbider psychischer Störungen im notwendigen Maße sicherzustellen und andrerseits um neue Therapien zu entwickeln. Zusammenfassend schreibt die ICD-11 die Diagnosen und diagnostischen Kriterien von Sprech- oder Sprachstörungen aus der ICD-10 und dem DSM5 in weiten Teilen fort. Neu ist - analog zum DSM-5 - die Aufnahme der Diagnose 6A01.22: Soziale (pragmatische) Kommunikationsstörung, deren diagnostische Validität in Zukunft und Abgrenzung zur Autismus-Spektrum-Störung noch besser aufgeklärt werden muss (Freitag, 2020). Die (Weiter-)Entwicklung von geeigneten diagnostischen Instrumenten insbesondere hinsichtlich der Aspekte der linguistischen Feindifferenzierung sowie die Entwicklung von normierten Instrumenten für Kinder jenseits des Vorschulalters und für Kinder mit DaZ ist ebenfalls essenziell, um Sprech- und Sprachstörungen korrekt diagnostizieren zu können.

\section{Literatur}

American Psychiatric Association. (2020). Diagnostische Kriterien DSM-5: Deutsche Ausgabe herausgegeben von Peter Falkai und Hans-Ulrich Wittchen, mitherausgegeben von Manfred Döpfner, Wolfgang Gaebel, Wolfgang Maier, Winfried Rief, Henning Saß und Michael Zaudig (2., korrigierte Aufl.). Göttingen: Hogrefe.

Barnett, C.P. \& van Bon, B.W.M. (2015). Monogenic and chromosomal causes of isolated speech and language impairment. Journal of Medical Genetics, 52, 719-729.
Bishop, D.V.M., Snowling, M.J., Thompson, P.A. \& Greenhalgh, T. (2016). CATALISE: A multinational and multidisciplinary Delphi consensus study. Identifying language impairments in children. Plos One, 11, e0158753.

Bishop, D.V.M., Snowling, M.J., Thompson, P.A. \& Greenhalgh, T. (2017). Phase 2 of CATALISE: A multinational and multidisciplinary Delphi consensus study of problems with language development: Terminology. Journal of Child Psychology and Psychiatry, and Allied Disciplines, 58, 1068-1080.

Cohan, S.L., Chavira, D.A., Shipon-Blum, E., Hitchcock, C., Roesch, S.C. \& Stein, M.B. (2008). Refining the classification of children with selective mutism: A latent profile analysis. Journal of Clinical Child and Adolescent Psychology, 37, 770-784.

Freitag, C.M. (2020). Von den tiefgreifenden Entwicklungsstörungen in ICD-10 zur Autismus-Spektrum-Störung in ICD-11. Zeitschrift für Kinder- und Jugendpsychiatrie und Psychotherapie, epub.

Grimm, A. \& Schulz, P. (2014). Specific language impairment and early second language acquisition: The risk of over- and underdiagnosis. Child Indicators Research, 7, 821-841.

Grimm, H. (2015). SETK 3-5: Sprachentwicklungstest für drei- bis fünfährige Kinder (3;0-5;11 Jahre) (3., überarb. und neu normierte Aufl.). Göttingen: Hogrefe.

Grimm, H. (2016). SETK-2: Sprachentwicklungstest für zweijährige Kinder (2., überarb. und neu normierte Aufl.). Göttingen: Hogrefe.

Hildebrand, M.S., Jackson, V.E., Scerri, T.S., van Reyk, O., Coleman, M., Braden, R. O. et al. (2020). Severe childhood speech disorder: Gene discovery highlights transcriptional dysregulation. Neurology, 94, e2148-e2167.

Kauschke, C. \& Vogt, S. (2019). Positionspapier zur Terminologie und Klassifikation von Sprachentwicklungsstörungen. Logos, 27, 174-181.

Nickisch, A., Gross, M., Schönweiler, R., Uttenweiler, V., am Zehnhoff-Dinnesen, A. am Berger, R. et al. (2000). Auditive Verarbeitungs- und Wahrnehmungsstörungen: Konsensus-Statement der Deutschen Gesellschaft für Phoniatrie und Pädaudiologie. HNO, 55, 61-72.

Norbury, C.F. (2014). Practitioner review: Social (pragmatic) communication disorder conceptualization, evidence and clinical implications. Journal of Child Psychology and Psychiatry, and Allied Disciplines, 55, 204-216.

Remschmidt, H., Schmidt, M.H. \& Poustka, F. (2017). Multiaxiales Klassifikationsschema für psychische Störungen des Kindesund Jugendalters nach ICD-10: Mit einem synoptischen Vergleich von ICD-10 und DSM-5 (7., akt. Aufl.). Bern: Hogrefe.

Reuner, G. \& Rosenkranz, J. (2014). Bayley Scales of Infant and Toddler Development - Third Edition - Deutsche Fassung. Frankfurt: Pearson.

Schulz, P. (2013). Sprachdiagnostik bei mehrsprachigen Kindern. Sprache - Stimme - Gehör, 37, 191-195.

Schulz, P. \& Grimm, A. (2012). Spracherwerb. In H. Drügh, S. Komfort-Hein, A. Kraß, C. Meier, G. Rohowski, R. Seidel et al. (Hrsg.), Germanistik (S. 155-172). Stuttgart: J.B. Metzler.

Schulz, P. \& Tracy, R. (2011). Linguistische Sprachstandserhebung Deutsch als Zweitsprache: LiSe-DaZ. Göttingen: Hogrefe.

Teufel, K., Wilker, C., Valerian, J. \& Freitag, C. M. (2017). A-FFIP - Autismusspezifische Therapie im Vorschulalter (Psychotherapie: Manuale). Berlin: Springer.

Verhoef, E., Shapland, C.Y., Fisher, S. E., Dale, P.S. \& St Pourcain, B. (2021). The developmental genetic architecture of vocabulary skills during the first three years of life: Capturing emerging associations with later-life reading and cognition. PLoS Genetics, 17, e1009144.

Voet Cornelli, B., Schulz, P. \& Tracy, R. (2013). Sprachentwicklungsdiagnostik bei Mehrsprachigkeit. Monatsschrift Kinderheilkunde, 161, 911-917. 
Wellnitz, S.A.C., Kästel, I., Vllasaliu, L., Cholemkery, H., Freitag, C.M. \& Bast, N. (2021). The Revised Children's Communication Checklist-2 (CCC-R): Factor structure and psychometric evaluation. Autism Research, epub.

Weltgesundheitsorganisation. (1994). ICD-10: Internationale statistische Klassifikation der Krankheiten und verwandter Gesundheitsprobleme. 10. Revision: Band I - Systematisches Verzeichnis. Version 1.0, Stand August 1994. Berlin: Springer.

\section{Historie}

Manuskript eingereicht: 18.03.2021

Nach Revision angenommen: 10.06.2021

Onlineveröffentlichung: 16.07.2021

\section{Interessenkonflikt}

Es bestehen keine Interessenskonflikte.

\section{Förderung}

Open-Access-Veröffentlichung ermöglicht durch die GoetheUniversität Frankfurt am Main.

\section{Prof. Dr. Christine M. Freitag}

Klinik für Psychiatrie, Psychosomatik und Psychotherapie des

Kindes- und Jugendalters, Autismus-Therapie- und Forschungszentrum

Universitätsklinikum Frankfurt, Goethe-Universität Deutschordenstr. 50

60528 Frankfurt am Main

Deutschland

c.freitag@em.uni-frankfurt.de 